Article

\title{
Analytical Solution of Urysohn Integral Equations by Fixed Point Technique in Complex Valued Metric Spaces
}

\author{
Hasanen A. Hammad ${ }^{1}(\mathbb{D})$ and Manuel De la Sen ${ }^{2, *}$ \\ 1 Department of Mathematics, Faculty of Science, Sohag University, Sohag 82524, Egypt; \\ hassanein_hamad@science.sohag.edu.eg \\ 2 Institute of Research and Development of Processes University of the Basque Country, \\ 48940 Leioa, Bizkaia, Spain \\ * Correspondence: manuel.delasen@ehu.eus
}

Received: 18 July 2019; Accepted: 10 September 2019; Published: 15 September 2019

\begin{abstract}
The purpose of this article is to introduce a fixed point result for a general contractive condition in the context of complex valued metric spaces. Also, some important corollaries under this contractive condition are obtained. As an application, we find a unique solution for Urysohn integral equations, and some illustrative examples are given to support our obtaining results. Our results extend and generalize some other known results in the literature.
\end{abstract}

Keywords: single-valued mappings; complex valued metric spaces; common fixed point; nonlinear integral equations

MSC: 47H09; 47H10

\section{Introduction}

The fixed point theorem, generally known as the Banach contraction principle, appeared in explicit form in Banach thesis in 1922. Fixed point theory is very famous because of its variety of applications in numerous areas, such as computer science, engineering, economics, etc. The contractive type conditions play an important role in the fixed point theory. Many researchers have extended and generalized this principle because it is the heart of fixed point theory (see, for example, the works of the authors of [1-7]).

The complex valued metric spaces is more general than ordinary metric spaces. According to this concept, a number of articles related to fixed point theory and it's application are presented (see, for example, [8-22]).

The aim of this paper is to prove a fixed point theorem in complex valued metric spaces under contractive condition for single-valued mappings. Moreover, we give a result of existence and uniqueness for solutions of a nonlinear system of integral equations. Finally, we give some explained examples to strengthen our results.

\section{Preliminaries and Known Results}

This section is prepared to discuss some known notations and definitions that will be used later. Suppose that $\mathbb{C}$ is the set of complex numbers and $z_{1}, z_{2} \in \mathbb{C}$. Define a partial order $\preceq$ on $\mathbb{C}$ :

$$
z_{1} \preceq z_{2} \text { iff } \operatorname{Re}\left(z_{1}\right) \leq \operatorname{Re}\left(z_{2}\right) \text { and } \operatorname{Im}\left(z_{1}\right) \leq \operatorname{Im}\left(z_{2}\right) \text {. }
$$


So, $z_{1} \preceq z_{2}$ if one of the following conditions hold.

$$
\begin{aligned}
& \text { (i) } \operatorname{Im}\left(z_{1}\right)<\operatorname{Im}\left(z_{2}\right) \text { and } \operatorname{Re}\left(z_{1}\right)=\operatorname{Re}\left(z_{2}\right), \\
& \text { (ii) } \operatorname{Im}\left(z_{1}\right)=\operatorname{Im}\left(z_{2}\right) \text { and } \operatorname{Re}\left(z_{1}\right)<\operatorname{Re}\left(z_{2}\right), \\
& \text { (iii) } \operatorname{Im}\left(z_{1}\right)<\operatorname{Im}\left(z_{2}\right) \text { and } \operatorname{Re}\left(z_{1}\right)<\operatorname{Re}\left(z_{2}\right), \\
& \text { (iv) } \operatorname{Im}\left(z_{1}\right)=\operatorname{Im}\left(z_{2}\right) \text { and } \operatorname{Re}\left(z_{1}\right)=\operatorname{Re}\left(z_{2}\right) .
\end{aligned}
$$

Here, we write $z_{1} \precsim z_{2}$ if $z_{1} \neq z_{2}$ and one of conditions (i), (ii), and (iii) is satisfied and if only (iii) is satisfied, we write $z_{1} \prec z_{2}$.

Definition 1 ([8]). Let $\Omega \neq \varnothing$. A mapping $\omega: \Omega \times \Omega \rightarrow \mathbb{C}$ is said to a complex valued metric on $\Omega$ if the following conditions holds for all $\kappa, \mu, \tau \in \Omega$,

$\left(C M_{1}\right) 0 \precsim \omega(\kappa, \mu)$ and $\omega(\kappa, \mu)=0 \Leftrightarrow \kappa=\mu$;

$\left(C M_{2}\right) \omega(\kappa, \mu)=\omega(\mu, \kappa)$;

$\left(C M_{3}\right) \omega(\kappa, \mu) \precsim \omega(\kappa, \tau)+\omega(\tau, \mu)$.

Then $\Omega$ is called a complex valued metric on $\Omega$ and $(\Omega, \omega)$ is called a complex valued metric space.

For some examples of complex valued metric spaces see the works by the authors of $[8,9,14,17]$. Now, we state two examples not mentioned above.

Example 1. Let $\Omega=\mathbb{C}$ be a set of complex number. Define a distance $\omega^{\prime}: \Omega \times \Omega \rightarrow \Omega$, by

$$
\omega^{\prime}\left(z_{1}, z_{2}\right)=\omega\left(\kappa_{1}, \kappa_{2}\right)+i \omega\left(\mu_{1}, \mu_{2}\right),
$$

for all $z_{1}, z_{2} \in \Omega$, where $z_{1}=\left(\kappa_{1}, \mu_{1}\right)$ and $z_{2}=\left(\kappa_{2}, \mu_{2}\right)$. Then, $\left(\Omega, \omega^{\prime}\right)$ is a complex valued metric space provided that $(\Omega, \omega)$ is too.

Example 2. Let $\Omega=\mathbb{C}$ be a set of complex number. Define the distance $\omega: \Omega \times \Omega \rightarrow \Omega$ by

$$
\omega\left(z_{1}, z_{2}\right)=\left(\left(\kappa_{1}-\kappa_{2}\right)^{2}+i\left(\mu_{1}-\mu_{2}\right)^{2}\right)^{\frac{1}{2}},
$$

where $z_{1}=\kappa_{1}+i \mu_{1}$ and $z_{2}=\kappa_{2}+i \mu_{2}$. Then, $(\Omega, \omega)$ is a complex valued metric space.

Definition 2 ([8]). Let $(\Omega, \omega)$ be a complex valued metric space. Then:

(i) A sequence $\left\{\kappa_{n}\right\}$ in $\Omega$ is said to be converge to $\kappa \in \Omega$ if for every $0 \prec \varepsilon \in \mathbb{C}$ there exists $N \in \mathbb{N}$ such that $\omega\left(\kappa_{n}, \kappa\right) \prec \varepsilon \forall n>N$. We denote this by $\lim _{n \rightarrow \infty} \kappa_{n}=\kappa$ or $\kappa_{n} \rightarrow \kappa$ as $n \rightarrow \infty$.

(ii) If for every $0 \prec \varepsilon \in \mathbb{C}$ there exists $N \in \mathbb{N}$ such that $\omega\left(\kappa_{n}, \kappa_{n+m}\right) \prec \varepsilon$ for all $n>N, m \in N$, then $a$ sequence $\left\{\kappa_{n}\right\}$ is called a Cauchy sequence in $\Omega$.

(iii) If every Cauchy sequence is convergent, then $(\Omega, \omega)$ is called a complete complex valued metric space.

Lemma 1 ([8]). Let $\left\{\kappa_{n}\right\}$ be a sequence in $(\Omega, \omega)$. It is said that $\left\{\kappa_{n}\right\}$ converges to $\kappa$ iff $\left|\omega\left(\kappa_{n}, \kappa\right)\right| \rightarrow 0$ as $n \rightarrow \infty$.

Lemma 2 ([8]). Let $\left\{\kappa_{n}\right\}$ be a sequence in $(\Omega, \omega)$. It is said that $\left\{\kappa_{n}\right\}$ in $\Omega$ is a Cauchy sequence if $\left|\Omega\left(\kappa_{n}, \kappa_{n+m}\right)\right| \rightarrow 0$ as $n \rightarrow \infty$, where $m \in N$.

\section{Main Result}

We introduce our first result. 
Theorem 1. Let $A$ and $B$ be self-mappings on a complete complex valued metric space $(\Omega, \omega)$, such that

$$
\omega(A \kappa, B \mu) \preceq \alpha \Theta(\kappa, \mu),
$$

$\forall \kappa, \mu \in \Omega$, where $\alpha \in(0,1)$,

$$
\Theta(\kappa, \mu)=\max \left\{\omega(\kappa, y), \frac{\omega(\kappa, A \kappa) \omega(\mu, B \mu)}{1+\omega(\kappa, \mu)}, \frac{\omega(\kappa, B \mu) \omega(\mu, A \kappa)}{1+\omega(\kappa, \mu)}\right\} .
$$

Then there exists a unique common fixed point of the pair mappings $(A, B)$.

Proof. Let $\kappa_{\circ} \in \Omega$ be an arbitrary point. Define a sequence $\left\{\kappa_{n}\right\}$ as follows.

$$
\kappa_{2 n+1}=A \kappa_{2 n} \text { and } \kappa_{2 n+2}=B \kappa_{2 n+1}, n=0,1,2, \ldots
$$

Then, by Equations (1) and (2), we get

$$
\begin{aligned}
& \omega\left(\kappa_{2 n+1}, \kappa_{2 n+2}\right)=\omega\left(A \kappa_{2 n}, B \kappa_{2 n+1}\right) \\
\precsim & \alpha \max \left\{\omega\left(\kappa_{2 n}, \kappa_{2 n+1}\right), \frac{\omega\left(\kappa_{2 n}, A \kappa_{2 n}\right) \omega\left(\kappa_{2 n+1}, B \kappa_{2 n+1}\right)}{1+\omega\left(\kappa_{2 n}, \kappa_{2 n+1}\right)}, \frac{\omega\left(\kappa_{2 n}, B \kappa_{2 n+1}\right) \omega\left(\kappa_{2 n+1}, A \kappa_{2 n}\right)}{1+\omega\left(\kappa_{2 n}, \kappa_{2 n+1}\right)}\right\} \\
\precsim & \alpha \max \left\{\omega\left(\kappa_{2 n}, \kappa_{2 n+1}\right), \frac{\omega\left(\kappa_{2 n}, \kappa_{2 n+1}\right) \omega\left(\kappa_{2 n+1}, \kappa_{2 n+2}\right)}{1+\omega\left(\kappa_{2 n}, \kappa_{2 n+1}\right)}, \frac{\omega\left(\kappa_{2 n}, \kappa_{2 n+2}\right) \omega\left(\kappa_{2 n+1}, \kappa_{2 n+1}\right)}{1+\omega\left(\kappa_{2 n}, \kappa_{2 n+1}\right)}\right\} \\
\precsim & \alpha \max \left\{\omega\left(\kappa_{2 n}, \kappa_{2 n+1}\right), \omega\left(\kappa_{2 n+1}, \kappa_{2 n+2}\right)\right\} .
\end{aligned}
$$

If $\max \left\{\omega\left(\kappa_{2 n}, \kappa_{2 n+1}\right), \omega\left(\kappa_{2 n+1}, \kappa_{2 n+2}\right)\right\}=\omega\left(\kappa_{2 n+1}, \kappa_{2 n+2}\right)$, then

$$
\omega\left(\kappa_{2 n+1}, \kappa_{2 n+2}\right) \preceq \alpha \omega\left(\kappa_{2 n+1}, \kappa_{2 n+2}\right) ，
$$

Since $\alpha<1$ and both distances in the left and right-hand sides are identical this equation is not possible unless both distances are zero. So

$$
\omega\left(\kappa_{2 n+1}, \kappa_{2 n+2}\right) \precsim \alpha \omega\left(\kappa_{2 n}, \kappa_{2 n+1}\right) .
$$

Similarly, we can obtain that

$$
\omega\left(\kappa_{2 n+2}, \kappa_{2 n+3}\right) \precsim \alpha \omega\left(\kappa_{2 n+1}, \kappa_{2 n+2}\right) .
$$

From Equations (3) and (4) for all $n=0,1,2, \ldots$, we can write

$$
\omega\left(\kappa_{n+1}, \kappa_{n+2}\right) \precsim \alpha \omega\left(\kappa_{n}, \kappa_{n+1}\right) \precsim \ldots \precsim \alpha^{n+1} \omega\left(\kappa_{\circ}, \kappa_{1}\right) .
$$

So, for $m>n$,

$$
\begin{aligned}
\omega\left(\kappa_{n}, \kappa_{m}\right) & \precsim \omega\left(\kappa_{n}, \kappa_{n+1}\right)+\omega\left(\kappa_{n+1}, \kappa_{n+2}\right)+\ldots+\omega\left(\kappa_{m-1}, \kappa_{m}\right) \\
& \precsim\left(\alpha^{n}+\alpha^{n+1}+\ldots+\alpha^{m-1}\right) \omega\left(\kappa_{\circ}, \kappa_{1}\right) \\
& \precsim\left(\frac{\alpha^{n}}{1-\alpha}\right) \omega\left(\kappa_{\circ}, \kappa_{1}\right) .
\end{aligned}
$$

So,

$$
\left|\omega\left(\kappa_{n}, \kappa_{m}\right)\right| \precsim\left(\frac{\alpha^{n}}{1-\alpha}\right)\left|\omega\left(\kappa_{\circ}, \kappa_{1}\right)\right| \rightarrow 0 \text { as } n \rightarrow \infty \text {. }
$$


Therefore, $\left\{\kappa_{n}\right\}$ is a Cauchy sequence in $\Omega$. Since $\Omega$ is complete, then there exists $u \in \Omega$, such that $\kappa_{n} \rightarrow u$. If $A$ and $B$ are not continuous, then $u=A u$, unlike that $\omega(u, A u)=z>0$ and one gets

$$
\begin{aligned}
z & \precsim \omega\left(u, \kappa_{2 l+2}\right)+\omega\left(A u, \kappa_{2 l+2}\right) \\
& \precsim \omega\left(u, \kappa_{2 l+2}\right)+\omega\left(A u, B \kappa_{2 l+1}\right) \\
& \precsim \omega\left(u, \kappa_{2 l+2}\right)+\alpha \max \left\{\omega\left(u, \kappa_{2 l+1}\right), \frac{\omega(u, A u) \omega\left(\kappa_{2 l+1}, B \kappa_{2 l+1}\right)}{1+d\left(u, \kappa_{2 l+1}\right)}, \frac{\omega\left(u, B \kappa_{2 l+1}\right) \omega\left(\kappa_{2 l+1}, A u\right)}{1+\omega\left(u, \kappa_{2 l+1}\right)}\right\} \\
& \precsim \omega\left(u, x_{2 l+2}\right)+\alpha \max \left\{\omega\left(u, \kappa_{2 l+1}\right), \frac{\omega(u, A u) \omega\left(\kappa_{2 l+1}, \kappa_{2 l+2}\right)}{1+\omega\left(u, \kappa_{2 l+1}\right)}, \frac{\omega\left(u, \kappa_{2 l+2}\right) \omega\left(\kappa_{2 l+1}, A u\right)}{1+\omega\left(u, \kappa_{2 l+1}\right)}\right\} \\
& \precsim \omega\left(u, \kappa_{2 l+2}\right)+\alpha \max \{0,0, z\} \\
& \precsim \omega\left(u, x_{2 l+2}\right)+\alpha z .
\end{aligned}
$$

This yields,

$$
|z| \leq\left|\omega\left(u, \kappa_{2 l+2}\right)\right|+\alpha|z|
$$

That is, $|z|=0$, is a contradiction, and hence $u=A u$. It follows, similarly, that $u=B u$.

If $A$ and $B$ are continuous, i.e., the continuity of $A$, yields

$$
u=\lim _{n \rightarrow \infty} \kappa_{2 n+2}=\lim _{n \rightarrow \infty} A \kappa_{2 n+1}=A \lim _{n \rightarrow \infty} \kappa_{2 n+1}=A u
$$

Similarly, $u=B u$. Hence the pair $(A, B)$ has a common fixed point.

Uniqueness. Suppose that $q \in \Omega$ is a another common fixed point of the nonlinear self-mappings $A$ and $B$. Then,

$$
\begin{aligned}
\omega(u, q) & =\omega(A u, B q) \\
& \precsim \alpha \max \left\{\omega(u, q), \frac{\omega(u, A u) \omega(q, B q)}{1+\omega(u, q)}, \frac{\omega(u, B q) \omega(q, A u)}{1+\omega(u, q)}\right\} \\
& \precsim \alpha d(u, q) .
\end{aligned}
$$

This implies that $u=q$, this completes the proof.

The following example support Theorem 1.

Example 3. Let $\Omega=[0, \infty)$ define the distance $\omega: \Omega \times \Omega \rightarrow \mathbb{C}$ by

$$
\omega(\kappa, \mu)=i|\kappa-\mu|
$$

It is clear that $(\Omega, \omega)$ is a complete complex valued metric space. We define the two self-mappings $A$ and $B$ as

$$
A \kappa=2 \kappa^{2}-1 \text { and } B \kappa=(2-\kappa)^{2} .
$$

Then the contractive condition Equation (1) is satisfied, indeed for $\kappa=\frac{1}{3}$ and $\mu=3$, we can write by the simple calculations of

and

$$
\omega(A \kappa, B \mu)=\frac{16}{9} i
$$

$$
\Theta(\kappa, \mu)=\max \left\{\frac{8}{3} i, \frac{-40}{3+8 i}, \frac{-68}{9(3+8 i)}\right\}=\frac{8}{3} i .
$$

So,

$$
\frac{16 i}{9} \precsim \alpha \frac{8 i}{3}
$$


Therefore, the conditions of Theorem 1 are verified with $\alpha=\frac{2}{3}<1$, and $1 \in \Omega$ is a unique common fixed point of $A$ and $B$.

If we take $A=B$ in the above theorem we have the following immediate consequences.

Corollary 1. Suppose that $A$ is a self-mapping on a complete complex valued metric space $(\Omega, \omega)$, such that

$$
\omega(A \kappa, A \mu) \precsim \alpha \Theta(\kappa, \mu),
$$

for all $\kappa, \mu \in \Omega$, where $0<\alpha<1$ and

$$
\Theta(\kappa, \mu)=\max \left\{\omega(\kappa, \mu), \frac{\omega(\kappa, A \kappa) \omega(\mu, A \mu)}{1+\omega(\kappa, \mu)}, \frac{\omega(\kappa, A \mu) \omega(\mu, A \kappa)}{1+\omega(\kappa, \mu)}\right\} .
$$

Then, in $\Omega$, a mapping A has a unique fixed point.

To justify the requirements of Corollary 1 , we present the following example.

Example 4. Let $\Omega=[0, \infty)$ and $\omega: \Omega \times \Omega \rightarrow \mathbb{C}$ be a mapping defined by

$$
\omega(\kappa, \mu)=|\kappa-\mu|+i|\kappa-\mu| \text {. }
$$

Clearly $(\Omega, \omega)$ is a complete complex valued metric space. Define a self-mapping A by

$$
A \kappa=\frac{2}{\pi} \sin ^{-1} \kappa
$$

To verify the contractive condition of Corollary 1 , we take $\kappa=\frac{1}{2}$ and $\mu=\frac{\sqrt{3}}{2}$, one can write by the simple calculations of

$$
\omega(A \kappa, A \mu) \simeq 0.1667(1+i) .
$$

and

$$
\Theta(\kappa, \mu) \simeq \max \{0.3660(1+i), 0.0483 i, 0.1301 i\} \simeq 0.3660(1+i) .
$$

So,

$$
0.1667(1+i) \precsim \alpha 0.3660(1+i) .
$$

Therefore, all conditions of Corollary 1 are satisfied with $\alpha \simeq 0.4555<1$ and $A$ has a unique fixed point $1 \in \Omega$.

Corollary 2. Consider $(\Omega, \omega)$ is a complete complex valued metric space and $A: \Omega \rightarrow \Omega$, there exists $n \in \mathbb{N}$ such that

$$
\omega\left(A^{n} \kappa, A^{n} \mu\right) \precsim \alpha \Theta(\kappa, \mu)
$$

$\forall \kappa, \mu \in \Omega$, where $0<\alpha<1$,

$$
\Theta(\kappa, \mu)=\max \left\{\omega(\kappa, \mu), \frac{\omega\left(\kappa, A^{n} \kappa\right) \omega\left(\mu, S^{n} \mu\right)}{1+\omega(\kappa, \mu)}, \frac{\omega\left(\kappa, A^{n} \mu\right) \omega\left(\mu, A^{n} \kappa\right)}{1+\omega(\kappa, \mu)}\right\} .
$$

Then A possesses a unique fixed point.

Proof. By Corollary 1, we obtain $v \in \Omega$ such that

$$
A^{n} v=v .
$$


So,

$$
\begin{aligned}
\omega(A v, v) & =\omega\left(A A^{n} v, A^{n} v\right)=\omega\left(A^{n} A v, A^{n} v\right) \\
& \precsim \alpha \max \left\{\omega(A v, v), \frac{\omega\left(A v, A^{n} A v\right) \omega\left(v, A^{n} v\right)}{1+\omega(A v, v)}, \frac{\omega\left(A v, A^{n} v\right) \omega\left(v, A^{n} A v\right)}{1+\omega(A v, v)}\right\} \\
& \precsim \alpha \max \left\{\omega(A v, v), \frac{\omega\left(A v, A A^{n} v\right) \omega\left(v, A^{n} v\right)}{1+\omega(A v, v)}, \frac{\omega\left(A v, A^{n} v\right) \omega\left(v, A A^{n} v\right)}{1+\omega(A v, v)}\right\} \\
& =\alpha \omega(A v, v) .
\end{aligned}
$$

Therefore, the result is follows.

Now we shall give a numerical example to show the validity of Corollary 2.

Example 5. Let $\Omega=C([0,2], \mathbb{R}), b>0$,

$$
\begin{aligned}
N_{\kappa \mu} & =\max _{t \in[0,2]}|\kappa(t)-\mu(t)|, \\
\omega(\kappa, \mu) & =N_{\kappa \mu} \sqrt[3]{1+b^{3}} e^{i \cot ^{-1} b} .
\end{aligned}
$$

Define $A: \Omega \rightarrow \Omega$ by

$$
A \kappa(t)=1+3 \int_{0}^{t} u^{2} \kappa(u) d u, t \in[0,2] .
$$

For every $\kappa, \mu \in \Omega$, we have

$$
\begin{aligned}
\omega(A \kappa, A \mu) & =N_{A \kappa A \mu} \sqrt[3]{1+b^{3}} e^{i \cot ^{-1} b}=\max _{t \in[0,2]}|A \kappa(t)-A \mu(t)| \sqrt[3]{1+b^{3}} e^{i \cot ^{-1} b} \\
& \precsim 3 \int_{0}^{2} \max _{t \in[0,2]}|\kappa(u)-\mu(u)| u^{2} \sqrt[3]{1+b^{3}} e^{i \cot ^{-1} b} d u \\
& \precsim 8 \omega(\kappa, \mu) .
\end{aligned}
$$

Similarly,

$$
\omega\left(A^{n} \kappa, A^{n} \mu\right) \precsim \frac{8^{n}}{n !} \omega(\kappa, \mu) \precsim \frac{8^{n}}{n !} \Theta(\kappa, \mu),
$$

where,

$$
\frac{8^{n}}{n !} \simeq \begin{cases}295.894 & \text { If } n=10 \\ 26.906 & \text { If } n=15 \\ 1.185 & \text { If } n=19 \\ 0.474 & \text { If } n=20\end{cases}
$$

Thus, for $\alpha \simeq 0.474<1, n=20$, all conditions of Corollary 2 are verified, so A has a unique fixed point, which is a unique solution of the nonlinear integral equation

$$
\kappa(t)=1+3 \int_{0}^{t} u^{2} \kappa(u) d u, t \in[0,2],
$$

or the differential equation (initial value problem)

$$
\kappa^{\prime}(t)-3 \kappa^{2} t=0, t \in[0,2], t(0)=1 .
$$




\section{An Application to Urysohn Integral Type Equations}

This section is the main result of the paper, here we apply Theorem 1 to find a unique solution of the Urysohn integral type equations

$$
\left\{\begin{array}{l}
\kappa(t)=h(t)+\int_{a}^{b} \chi_{1}(t, s, \kappa(s)) d s \\
\mu(t)=h(t)+\int_{a}^{b} \chi_{2}(t, s, \mu(s)) d s
\end{array} .\right.
$$

where,

(i) $\kappa(t)$ and $\mu(t)$ are unknown variables for each $t \in[a, b], a>0$,

(ii) $h(t)$ is the deterministic free term defined for $t \in[a, b]$,

(iii) $\chi_{1}(t, s)$ and $\chi_{2}(t, s)$ are deterministic kernels defined for $t, s \in[a, b]$.

Let $\Omega=\left(C[a, b], \mathbb{R}^{n}\right), a>0$ and $\omega: \Omega \times \Omega \rightarrow \mathbb{R}^{n}$ defined by

$$
\omega(\kappa, \mu)=\sup _{t \in[a, b]}\|\kappa(t)-\mu(t)\|_{\infty} \sqrt[3]{1+b^{3}} e^{i \cot ^{-1} b},
$$

for all $\kappa, \mu \in \Omega, i=\sqrt{-1} \in \mathbb{C}$.

It's obvious that $\left(C[a, b], \mathbb{R}^{n},\|\cdot\|_{\infty}\right)$ is a complete complex valued metric space.

Next, we consider a system Equation (5) under the following conditions,

$\left(H_{1}\right) h(t) \in \Omega$,

$\left(H_{2}\right) \chi_{1}, \chi_{2}:[a, b] \times[a, b] \times \mathbb{R}^{n} \rightarrow \mathbb{R}^{n}$ are continuous functions satisfying

$$
\left|\chi_{1}(t, s, u(s))-\chi_{1}(t, s, v(s))\right| \precsim \frac{1}{(b-a) e^{a b}} \Theta(u, v),
$$

where,

$$
\Theta(u, v)=\max \left\{\omega(u, v), \frac{\omega(u, A u) \omega(v, B v)}{1+\omega(u, v)}, \frac{\omega(u, B v) \omega(v, A u)}{1+\omega(u, v)}\right\} .
$$

Next, we state and prove our main theorem of this section.

Theorem 2. System Equation (5) has a unique common solution provided that the conditions $\left(H_{1}\right)$ and $\left(H_{2}\right)$ are satisfied.

Proof. For $\kappa, \mu \in\left(C[a, b], \mathbb{R}^{n}\right)$ and $t \in[a, b]$, we define the continuous mappings $A, B: \Omega \rightarrow \Omega$ by

$$
\begin{aligned}
& A \kappa(t)=h(t)+\int_{a}^{b} \chi_{1}(t, s, \kappa(s)) d s, \\
& B \mu(t)=h(t)+\int_{a}^{b} \chi_{2}(t, s, \mu(s)) d s .
\end{aligned}
$$

By this, we have

$$
\begin{aligned}
|A \kappa(t)-B \mu(t)| & =\int_{a}^{b}\left|\chi_{1}(t, s, \kappa(s))-\chi_{2}(t, s, \mu(s))\right| d s \\
& \precsim \int_{a}^{b} \frac{1}{(b-a) e^{a b}}|\Theta(\kappa, \mu)| d s \\
& =\frac{1}{(b-a) e^{a b}} \int_{a}^{b} \frac{e^{-i \cot ^{-1} b}}{\sqrt[3]{1+b^{3}}}|\Theta(\kappa, \mu)| \sqrt[3]{1+b^{3}} e^{i \cot ^{-1} b} d s \\
& \precsim \frac{1}{(b-a) e^{a b}} \frac{e^{-i \cot ^{-1} b}}{\sqrt[3]{1+b^{3}}}\|\Theta(\kappa, \mu)\|_{\infty} \int_{a}^{b} d s \\
& =\frac{1}{e^{a b}} \frac{e^{-i \cot ^{-1} b}}{\sqrt[3]{1+b^{3}}}\|\Theta(\kappa, \mu)\|_{\infty} .
\end{aligned}
$$


This gives,

$$
\sqrt[3]{1+b^{3}}|A \kappa(t)-B \mu(t)| e^{-i \cot ^{-1} b} \precsim \frac{1}{e^{a b}}\|\Theta(\kappa, \mu)\|_{\infty},
$$

or, equivalently,

$$
\|A \kappa(t)-B \mu(t)\|_{\infty} \precsim \frac{1}{e^{a b}}\|\Theta(\kappa, \mu)\|_{\infty},
$$

or,

$$
\omega(A \kappa, B \mu) \precsim \alpha \Theta(\kappa, \mu) .
$$

So, the condition Equation (1) of Theorem 1 is satisfied with $0<\alpha=\frac{1}{e^{a b}}<1$, Therefore the system Equation (5) has a unique common solution on $\Omega$.

Finally, we verify all conditions of Theorem 2 by the following example.

Example 6. Let $\Omega=C([a, b], \mathbb{R})$ and the following nonlinear integral equation as the form

$$
\left\{\begin{array}{l}
\kappa(t)=e^{4 i t}+\int_{a}^{b}\left(\frac{e^{-\frac{1}{4}}}{4\left(t+\frac{i s}{1+i s}+\kappa(s)\right)}\right) d s \\
\mu(t)=e^{4 i t}+\int_{a}^{b}\left(\frac{e^{-\frac{1}{4}}}{4\left(t+\frac{i s}{1+i s}+\mu(s)\right)}\right) d s .
\end{array}\right.
$$

Problem Equation (6) is a special case of problem Equation (5), where $h(t)=e^{4 i t}$ and

$$
\chi_{j}\left(t, s, u_{j}(s)\right)=\left(\frac{e^{-\frac{1}{4}}}{4\left(t+\frac{i s}{1+i s}+u_{j}(s)\right)}\right), j=1,2 .
$$

It's obvious that $\left(H_{1}\right)$ is satisfied, for $\left(H_{2}\right)$, we get

$$
\begin{aligned}
\left|\chi_{1}(t, s, \kappa(s))-\chi_{2}(t, s, \mu(s))\right| & =\frac{1}{4} e^{-\frac{1}{4}}\left|\frac{\kappa(s)-\mu(s)}{\left(t+\frac{i s}{1+i s}+\kappa(s)\right)\left(t+\frac{i s}{1+i s}+\mu(s)\right)}\right| \\
& \precsim \frac{1}{4} e^{-\frac{1}{4}}|\kappa(s)-\mu(s)| .
\end{aligned}
$$

Therefore, $\left(H_{2}\right)$ is hold with $\alpha=\frac{1}{4} e^{-\frac{1}{4}}<1$ and $\Theta(\kappa, \mu)=|\kappa(s)-\mu(s)|$. By Theorem 2, the problem Equation (6) has a unique solution.

\section{Conclusions}

By changing the definition of real valued metric into complex valued metric, complex valued-metric spaces are considered as a generalization of ordinary metric spaces. This change is expected to bring wider applications of fixed point theorems. In this paper, we prove some fixed point theorem in complex valued metric spaces under contractive condition for single-valued mappings. Also, we find a unique solution of a nonlinear system of integral equations and we support our theoretical results by some explained examples.

Author Contributions: H.A.H contributed in conceptualization, investigation,methodology, validation and wrting the original draft; M.D.l.S. contributed in funding adquisition, methodology, project administration, supervision, validation, visualization, writing and editing. Both Aurhors agree and aprove the final version of this manuscript.

Funding: This research received funding from the Basque Government through project IT1207-19.

Acknowledgments: This work was supported in part by the Basque Government through project IT1207-19.

Conflicts of Interest: The authors declare no conflicts of interest. 


\section{References}

1. Arandjelović, I.; Radenović, Z.; Radenović, S. Boyd-Wong-type common fixed point results in cone metric spaces. Appl. Math. Comput. 2011, 217, 7167-7171. [CrossRef]

2. Boyd, D.W.; Wong, J.S.W. On nonlinear contractions. Proc. Am. Math. Soc. 1969, 20, 458-464. [CrossRef]

3. Dugundji, J.; Granas, A. Fixed Point Theory; Monograe Matematycne: Warsazawa, Poland, 1982.

4. Kirk, W.A.; Sems, B. Handbook of Metric Fixed Point Theory; Kluwer Academic Publishers: Iowa City, IA, USA; Newcastle, UK, 2001.

5. Rakotch, E. A note in contractive mappings. Proc. Am. Math. Soc. 1962, 13, 459-465. [CrossRef]

6. Rus, I.A. Generalized Contractions and Applications; Cluj University Press: Cluj-Napoca, Romania, 2001.

7. Tarafdar, E. An approach to fixed-point theorems on uniform spaces. Trans. Am. Math. Soc. 1974, 191, $209-225$. [CrossRef]

8. Azam, A.; Fisher, B.; Khan, M. Common fixed point theorems in complex valued metric spaces. Numer. Funct. Anal. Optim. 2011, 32, 243-253.

9. Bhatt, S.; Chaukiyal, S.; Dimri, R.C. A common fixed point theorem for weakly compatible maps in complex valued metric spaces. Int. J. Math. Sci. Appl. 2011, 1, 1385-1389.

10. Chandok, S.; Kumar, D. Some common fixed point results for rational type contraction mappings in complex valued metric spaces. J. Oper. 2013, 2013. [CrossRef]

11. Datta, S.K.; Ali, S. A common fixed point theorem under contractive condition in complex valued metric spaces. Int. J. Adv. Sci. Technol. Res. 2012, 2, 467-475.

12. Dubey, A.K. Complex valued $b$-metric spaces and common fixed point theorems under rational contractions. J. Complex Anal. 2016, 2016. [CrossRef]

13. Khan, S.U.; Arshad, M.; Nashine, H.K.; Nazam, M. Some common fixed points of generalized contractive mappings on complex valued metric spaces. J. Anal. Num. Theor. 2017, 5, 73-80. [CrossRef]

14. Manro, S. Some common fixed point theorems in complex valued metric space using implicit function. Int. J. Anal. Appl. 2013, 2, 62-70.

15. Mitra, T.A. Common coupled fixed point result in complex valued metric space for two mappings. Int. J. Curr. Res. 2015, 7, 19555-19559.

16. Nashine, H.K.; Imdad, M.; Hassan, M. Common fixed point theorems under rational contractions in complex valued metric spaces. J. Nonlinear Sci. Appl. 2014, 7, 42-50. [CrossRef]

17. Rouzkard, F.; Imdad, M. Some common fixed point theorems complex valued metric spaces. Comput. Math. Appl. 2012, 64, 1866-1874. [CrossRef]

18. Singh, N.; Singh, D.; Badal, A.; Joshi, V. Fixed point theorems in complex valued metric spaces. J. Egypt. Math. Soc. 2016, 24, 402-409. [CrossRef]

19. Sintunavarat, W.; Kumam, P. Generalized common fixed point theorems in complex valued metric spaces and applications. J. Inequal. Appl. 2012, 2012, 84. [CrossRef]

20. Sintunavarat, W.; Yeol, J.C.; Kumam, P. Urysohn integral equations approach by common fixed points in complex valued metric spaces. Adv. Differ. Equ. 2013, 2013, 49. [CrossRef]

21. Sitthikul, K.; Saejung, S. Some fixed point theorems in complex valued metric space. Fixed Point Theory Appl. 2012, 2012, 189. [CrossRef]

22. Verma, R.K.; Pathak, R.K. Common fixed point theorems using property (E.A.) in complex valued metric spaces. Thai J. Math. 2013, 11, 347-355.

(C) 2019 by the authors. Licensee MDPI, Basel, Switzerland. This article is an open access article distributed under the terms and conditions of the Creative Commons Attribution (CC BY) license (http://creativecommons.org/licenses/by/4.0/). 